\title{
Evaluation and interpretation of latent class modelling strategies to characterise dietary trajectories across early life: a longitudinal study from the Southampton women's survey
}

\author{
K.V. Dalrymple ${ }^{1,2}$, H.M. Inskip ${ }^{1,3}$, C. Vogel ${ }^{1,3}$, K.M. Godfrey ${ }^{1,3}$, J. Baird ${ }^{1,3}$, C. Cooper ${ }^{1,3}$ and \\ S.R. Crozier ${ }^{1}$ \\ ${ }^{1}$ MRC Lifecourse Epidemiology Unit, Southampton General Hospital, University of Southampton, Southampton, UK, \\ ${ }^{2}$ Department of Women and Children's Health, School of Life Course Sciences, King's College London, London, UK \\ and \\ ${ }^{3}$ NIHR Southampton Biomedical Research Centre, University of Southampton and University Hospital Southampton \\ NHS Foundation Trust, Southampton, UK
}

Researchers and public health experts are increasingly interested in modelling longitudinal data to determine relationships between early life exposures and future health outcomes. Such relationships are particularly relevant for dietary exposures where poor diet quality over a period of time may be associated with an increased risk of developing obesity. Previous longitudinal dietary intake analyses have estimated population means over time using mixed effect and average growth models ${ }^{(1,2)}$. However, classifying individuals into subgroups (latent classes) who follow similar trajectories over time is potentially more informative than previous analytical approaches. These trajectories describe longitudinal patterns of diet quality and could therefore help identify when and in whom to intervene. Although several methods are available for defining latent classes ${ }^{(3)}$, these have yet to be applied to longitudinal measures of dietary intake across early life.

The aim of this study was to provide an overview of two latent class methods: growth mixture models (GMM) and group-based trajectory modelling (GBTM) using the gllamm and traj Stata commands, respectively. GBTM and GMM were selected over other models because they are able to handle missing data and unevenly spaced assessments over time. GBTM also allows the user to define the shape of the individual trajectories (e.g. linear/quadratic). These methods were applied to data from 2963 mother-child dyads from the Southampton Women's Survey (SWS). In the SWS cohort continuous diet quality indices (DQIs) were derived using principal component analysis from an interviewer-administered food frequency questionnaire collected in the mother preconception and at 11 and 34 weeks' gestation, and in the child at ages 6 months, 12 months, 3, 6-7 and 8-9 years. To identify the optimal number of latent classes for the DQIs, we used a forward modelling approach, as advised by the GRoLTS-Checklist, from one to six classes ${ }^{(5)}$. Each model was assessed using the following criteria for classifying and interpreting latent class analyses: the Bayesian information criteria, probability of class assignment, the ratio of the odds of a correct classification, group membership, and relative entropy.

According to the classification criteria, the optimal model included 5-trajectories, with results from the GMM and GBTM being most similar for the 5-trajectory model (Spearman's correlation $=0.98$ ). Both methods characterised the dietary trajectories as stable with horizontal lines, and the five trajectories defined as low $(\mathrm{GMM}=4.1 \%, \mathrm{GBTM}=4.8 \%)$, low-medium $(23.0 \%, 22.7 \%)$, medium $(39.0 \%, 39.0 \%)$, medium-high $(27.4 \%, 27.9 \%)$ and high $(6.5 \%, 5.6 \%)$.

Although both methods provide a similar interpretation of dietary trajectories, we propose using GBTM command for modelling longitudinal dietary intake because GMM was computationally more intensive. Additionally, the findings of this study provide a rationale to promote dietary changes preconceptionally as the results show that diet quality tracks from pre-conception into childhood.

\section{References}

1. Winpenny EM, van Sluijs EMF, White M, et al. (2018) Int J Behav Nutr Phys Act 15 (86).

2. Goff LM, Huang P, Silva MJ, et al. (2019) Br J Nutr 121, 1069-1079.

3. van der Nest G, Passos VL, Candel MJJM, et al. (2020) Adv Life Course Res 43

4. van de Schoot R, Sijbrandij M, Winter SD, et al. (2017) Struc Equ Modeling 24(3), 451-467.

5. Lennon H, Kelly S, Sperrin M, et al. (2018) BMJ Open 8: e020683. 